\title{
OpticAlly OBSERVED MicrobUbBle COALESCENCE AND COLlAPSE
}

Michiel Postema*1,2 , Ayache Bouakaz ${ }^{1,2}$, Chien Ting Chin ${ }^{1,3}$ and Nico de Jong ${ }^{1,2}$

${ }^{1}$ Dept. of Experimental Echocardiography, Erasmus University Medical Center, Rotterdam, The Netherlands

${ }^{2}$ Interuniversity Cardiology Institute of the Netherlands (ICIN), Utrecht, The Netherlands

${ }^{3}$ Foundation for Fundamental Research on Matter (FOM), Utrecht, The Netherlands

Abstract - Understanding the mechanisms of microbubble destruction is needed for the development of ultrasound guided drug and gene delivery methods and for the improvement of diagnostic ultrasonic contrast agent (UCA) detection methods. We performed 482 experiments on the coalescence and collapse mechanisms of a soft- shelled and a hard-shelled contrast agent, by subjecting an experimental lipid-shelled UCA and the hard-shelled UCA Quantison ${ }^{\mathrm{TM}}$ to $500 \mathrm{kHz}$, high- pressured ultrasound (MI 1.0 ), and recording microscopic images of these events with a fastframing camera. Results showed that bubble fragmentation into smaller bubbles is the primary mechanism for lipid-shelled contrast microbubble destruction during the first cycles after ultrasound arrival. In $28 \%$ of our experimental events with a lipid-shelled UCA, we observed bubble coalescence. The coalescence mechanism was observed to be analog to the process desribed for larger gas bubbles. Repetitive coalescence and fragmentation was clearly recorded with a fast-framing camera. We also demonstrated the formation and collapse of large lipid-shelled bubbles and bubble clusters. Furthermore we showed that sonic cracking is feasible for the hard-shelled contrast agent Quantison $^{\mathrm{TM}}$. 


\section{INTRODUCTION}

Ultrasonic contrast agent (UCA) bubble destruction has been under investigation for its potential application in high MI imaging techniques and in drug and gene delivery. Recently Van Wamel et al. demonstrated the molecular uptake of ovary cells in vitro, induced by ultrasound, with and without the presence of contrast agent bubbles [1]. Kimmel presented his investigation on angiogenesis in vitro and in vivo in fish epidermis [2]. He concluded that bubble collapse and jet formation were responsible for the observed rupture and the enhanced permeability. The success of these two and other such new therapeutic techniques in drug and gene delivery is directly related to our understanding of bubble collapse and coalescence. Dayton et al. defined the following mechanisms of rapid bubble destruction: rapid destruction due to diffusion, fragmentation into smaller bubbles, and formation of a shell defect causing gas escape [3]. Takeuchi introduced the term sonic cracking for the latter mechanism [4]. Postema et al. observed bubble destruction, like De Jong et al. [5], and coalescence [6]. Chomas et al. noted that fragmentation occurs within $0.5 \mathrm{~ns}$ of peak compression [7]. Brennen stated that most unstable situations occur when the bubble is contracting and decelerating [8]. Moreover he related the forming of a re-entrant jet from a collapsing bubble, to the bubble shattering into fragments when the jet impacted the other side of the bubble surface. An optical image sequence of jet occurrence in a contrast microbubble, was demonstrated by Postema et al. [9]. Apparently water was propelled into the freely flowing microbubble.

We studied the destruction mechanisms of contrast agents by subjecting two different contrast agents to high-pressured ultrasound, and recording microscopic images of these events with a fast-framing camera. 


\section{EXPERIMENTAL METHODS}

Our experimental setup for taking pictures of insonified contrast bubbles was extensively described in [6]. A Ø $200 \mu \mathrm{m}$ capillary hose, through which contrast agent was flowing, was fixed in the focal area of a V389-SU $500 \mathrm{kHz}$ single-element transducer (Panametrics Inc., Waltham, MA) transducer. The optical observations of the insonified UCA were recorded with an Imacon 468 fast framing camera (DRS Hadland, Ltd., Tring, UK), operating at $3 \mathrm{MHz}$. In all observations, the ultrasonic wave arrived at the focal area between the first two frames. Seven frames were taken during ultrasound insonification, spanning a full ultrasound cycle. Each frame corresponded to an $88 \times 58 \mu \mathrm{m}^{2}$ area. UCA bubbles were insonified by 10 cycles of $500 \mathrm{kHz}$ ultrasound at acoustic pressures corresponding to mechanical indices between 0.9 and 1.2 .

We investigated the destruction mechanisms of a soft-shelled and a hard-shelled UCA. The softshelled agent was an experimental UCA, supplied by Bracco Research SA, Geneva, Switzerland. It consists of phospholipid-encapsulated gas bubbles ranging in diameter from 1 to $6 \mu \mathrm{m}$ with a median of $2 \mu \mathrm{m}$. Undiluted UCA ( $5 \mathrm{ml}$ of a $0.9 \% \mathrm{NaCl}$ dilution, added to a $25 \mathrm{mg}$ vial) was inserted through the capillary hose using either a syringe pressed by hand or a hose operated by a gravity fed or pumped infusion. The hard-shelled agent was Quantison ${ }^{\mathrm{TM}}$ (Quadrant Healthcare plc, Nottingham, UK). The shell consists of human serum albumin and ranges in thickness from 200 to $300 \mathrm{~nm}$ [10]. It has a narrow size distribution with a mean diameter of $3.2 \mu \mathrm{m} .0 .35 \mathrm{mg}$ Quantison ${ }^{\mathrm{TM}}$ was diluted in $5 \mathrm{ml}$ isotonic water. This dilution was poured into the container and covered with a microscopic cover slip. We performed 482 experiments with the experimental UCA, and 57 experiments with Quantison $^{\mathrm{TM}}$. 


\section{RESULTS AND DISCUSSION}

Coalescence - The bubble coalescence process was described by Dhainaut as follows [11]. First, two bubbles collide, e.g. when expanding. Upon contact, there is a flattening of the bubble surface contacts trapping part of the bubble shells. The shell parts separating the two bubbles drain until the separation reaches a critical thickness. An instability mechanism will result in rupture of the separation and the formation of a coalesced bubble. One hundred and thirty three of our experiments showed bubble coalescence clearly according to this mechanism. An example of the phenomenon is giving in Figure 1. The microbubble in the upper half at $t=-5 \mu \mathrm{s}$, apparently split into two parts during the first cycles of ultrasound, leaving two thin, banana-shaped parts in the frames at $0 \mu \mathrm{s}$ and $0.33 \mu$ s. At $0.66 \mu$ s and $0.99 \mu$ s the bubbles were still separated by a bubble wall. This separation had already disappeared at $1.32 \mu \mathrm{s}$, while the shape of the merged bubble changed from ellipsoid to almost circular. At $1.99 \mu$ s the bubble appeared circular, though hardly visible. Thus, the coalescence mechanism is analog to the process described by [11]. Bubble coalescence was only clearly observed with the experimental UCA, and not with Quantison ${ }^{\mathrm{TM}}$. The hard-shelled Quantison ${ }^{\mathrm{TM}}$ bubbles oscillated with too small excursions to be detected, as predicted by theory [12]. For the concentration used, the distances between the Quantison ${ }^{\mathrm{TM}}$ bubbles were much larger than the maximal excursions, and therefore bubble collision during the expansion phase may not occur. Due to coalescence, the experimental UCA microbubbles obtained sizes closer to resonance.

Fragmentation - We recorded 83 events demonstrating bubble fragmentation into smaller bubbles. An example is given in Figure 2. At $t=0 \mu$ s the two bubbles in the middle of the frame had already fragmented into six bubbles. During expansion phase they coalesced to one big, asymmetric bubble, visible at $t=0.99 \mu \mathrm{s}$. During compression $(t=1.65 \mu \mathrm{s})$ it fragmented into smaller bubbles. Such repetitive coalescing and fragmenting behavior was clearly recorded in 12 events. This suggests that the destruction behavior of contrast microbubbles is more complex than previously assumed. Bubble fragmentation was only observed with the experimental UCA, and not with Quantison ${ }^{\mathrm{TM}}$. We conclude that its bubble wall velocity and acceleration are too low to induce instabilities big enough for fission. In 2 events we clearly observed jets. We did not observe fragmentation in the same events however.

Sonic cracking - We observed the sonic cracking mechanism in 17 experiments, all of which were performed with Quantison $^{\mathrm{TM}}$. In total we recorded 38 different bubbles releasing free gas. Figure 3 shows an example of the sonic cracking phenomenon of two Quantison ${ }^{\mathrm{TM}}$ microbubbles. After 
cracking, the gas escaped. The cracked bubble in the upper right of the frames had already visible gas escape at $t=0.33 \mu \mathrm{s}$, whereas the cracked bubble in the lower right of the frames had the first visible sign of gas escape at $t=0.66 \mu \mathrm{s}$. On the recorded images the free gas expanded to up to five times the original diameter of a Quantison ${ }^{\mathrm{TM}}$ bubble. In all 57 experiments with Quantison ${ }^{\mathrm{TM}}$, the number of cracking bubbles per event is low compared to the number of bubbles present. The highest cracking ratio observed was 8 cracking bubbles on a total number of 31 . Tiny flaws in the bubble shell, may account for the reason why certain bubbles crack and others stay intact. The mechanism of sonic cracking itself is not yet understood.

Other mechanisms - Only 15 events were recorded that demonstrated other destruction mechanisms than fragmentation into smaller bubbles or sonic cracking, e.g. asymmetric collapse without fragmentation.

Since we were only looking at early cycles of the ultrasonic waves, this limits our conclusion that fission is the primary mechanism for bubble destruction. 


\section{CONCLUSIONS}

At before-mentioned acoustic frequency and pressures, we found bubble fragmentation into smaller bubbles the primary mechanism for lipid-shelled contrast microbubble destruction during the first cycles after ultrasound arrival. In $28 \%$ of our experimental events with a lipid-shelled UCA, we observed bubble coalescence. The coalescence mechanism was observed to be analog to the process desribed for larger gas bubbles. Due to coalescence, the experimental UCA microbubbles obtained sizes closer to resonance. Repetitive coalescence and fragmentation was clearly recorded with a fastframing camera. We demonstrated the formation and collapse of large lipid-shelled bubbles and bubble clusters. This coalescence and collapse mechanism of ultrasound contrast bubbles is analog to a previously presented mechanism on destructive properties of cavitation bubbles. We showed that sonic cracking is feasible for the hard-shelled contrast agent Quantison ${ }^{\mathrm{TM}}$. 
V. ACKNOWLEDGMENTS

This work has been supported by the Technology Foundation STW (RKG.5104). 


\section{REFERENCES}

[1] A. van Wamel, A. Bouakaz, F. ten Cate, N. de Jong, and J. Houtgraaf, "Effects of diagnostic ultrasound parameters on molecular uptake and cell viability," presented at 2nd ISTU, Seattle, 2002.

[2] E. Kimmel, "Micro-alterations and enhanced transport in cellular tissues exposed to therapeutic ultrasound," presented at 16th ISNA, Moscow, 2002.

[3] P. Dayton, K. Morgan, M. Allietta, A. Klibanov, G. Brandenburger, and K. Ferrara, "Simultaneous optical and acoustical observations of contrast agents," in Proc. IEEE Ultrason. Symp., 1997, pp. 1583-1591.

[4] Y. Takeuchi, "A sequence of microscopic image frames collected during insonification of a hardshell model contrast agent," IEEE Trans. Ultrason., Ferrorelect., Freq. Contr., vol. 46(4), cover page, 1999.

[5] N. de Jong, P. J. A. Frinking, A. Bouakaz, M. Goorden, T. Schuurmans, X. Jingping, and F. Mastik, "Optical imaging of contrast agent microbubbles in an ultrasound field with a $100-\mathrm{MHz}$ camera," Ultrasound Med. Biol., vol. 26, pp. 487-492, 2000.

[6] M. Postema, A. Bouakaz, C. T. Chin, and N. de Jong, "Real-time optical imaging of individual microbubbles in an ultrasonic field," in Proc. IEEE Ultrason. Symp., 2001, pp. 1679-1682.

[7] J. E. Chomas, P. Dayton, D. May, and K. Ferrara, "Threshold of fragmentation for ultrasonic contrast," J. Biomed. Opt., vol. 6, pp. 141-150, 2001.

[8] C. E. Brennen, "Fission of collapsing cavitation bubbles," presented at CAV 2001 4th Int. Symp. Cavitation, Pasadena, 2001.

[9] M. Postema, A. Bouakaz, and N. de Jong, "A sequence of microscopic image frames of a freely flowing contrast agent microbubble," IEEE Trans. Ultrason., Ferrorelect., Freq. Contr ., vol. 49(3), cover page, 2002.

[10] P. J. A. Frinking and N. de Jong, "Acoustic modeling of shell-encapsulated gas bubbles," Ultrasound Med. Biol., vol. 24, pp. 523-533, 1998.

[11] M. Dhainaut, "Literature study on observations andexperimentsoncoalescenceandbreakupof bubbles and drops," NTNU - Dept. of Chemical Engineering, Trondheim STF24 A02531, 2002. 
[12] N. de Jong, A. Bouakaz, and P. Frinking, "Basic acoustic properties of microbubbles," Echocardiography, vol. 19, pp. 229-240, 2002.

* Michiel Postema e-mail: postema@tch.fgg.eur.nl 


\section{List of Figures}

Figure 1: Lipid-shelled bubble coalescence

Figure 2: Lipid-shelled bubble fragmentation

Figure 3: Sonic cracking of two Quantison ${ }^{\mathrm{TM}}$ bubbles 


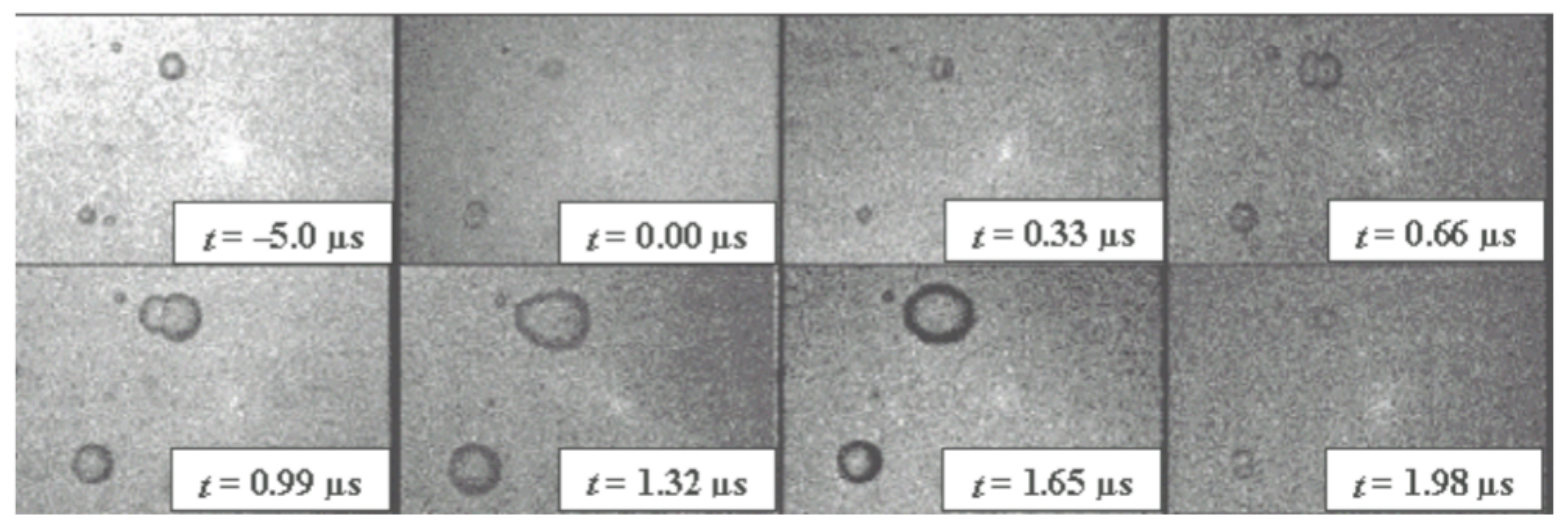

Figure 1: Lipid-shelled bubble coalescence

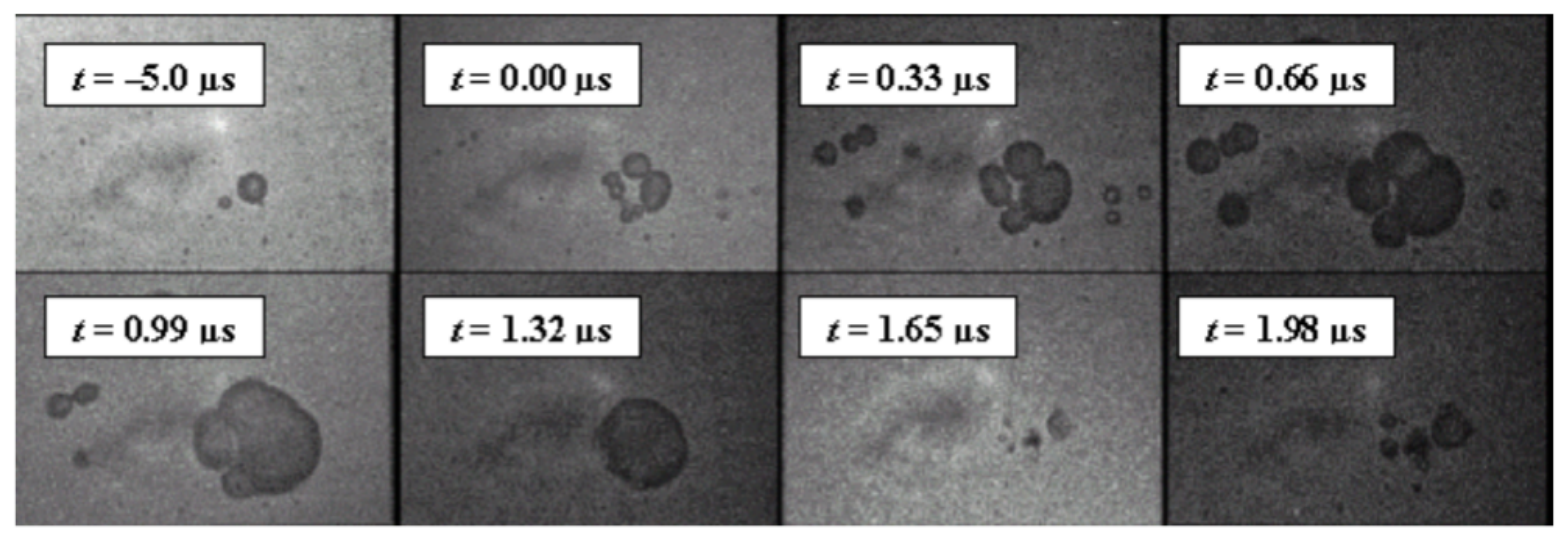

Figure 2: Lipid-shelled bubble fragmentation

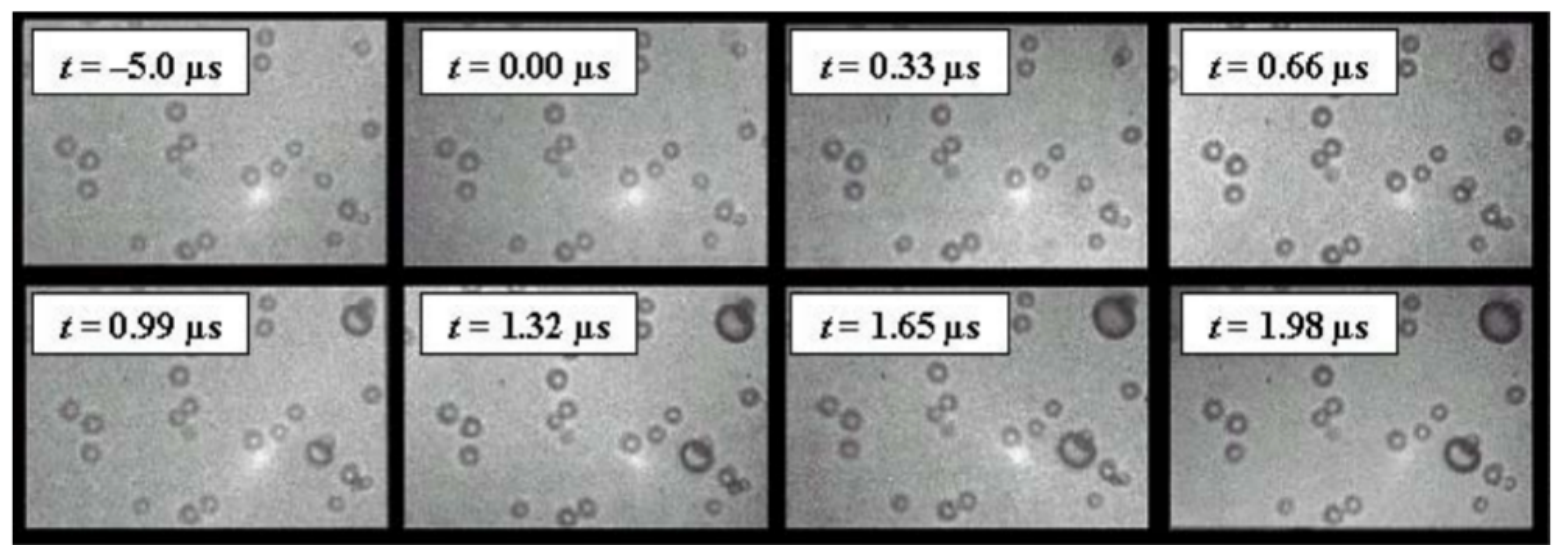

Figure 3: Sonic cracking of two Quantison ${ }^{\mathrm{TM}}$ bubbles 